\title{
High throughput sequencing of T-cell receptor repertoire using dry blood spots
}

\author{
Shang-Gin Wu ${ }^{1,2+}$, Wenjing Pan ${ }^{3,4+}{ }^{,}$Hongna Liu ${ }^{5}$, Miranda L. Byrne-Steele ${ }^{4}$, Brittany Brown ${ }^{4}$, Mollye Depinet ${ }^{4}$,
} Xiaohong $\mathrm{Hou}^{4}$, Jian $\mathrm{Han}^{3,4^{*}}$ and Song $\mathrm{Li}^{4^{*}}$

\begin{abstract}
Background: Immunology research, particularly next generation sequencing (NGS) of the immune T-cell receptor $\beta$ (TCRß) repertoire, has advanced progression in several fields, including treatment of various cancers and autoimmune diseases. This study aimed to identify the TCR repertoires from dry blood spots (DBS), a method that will help collecting real-world data for biomarker applications.
\end{abstract}

Methods: Finger-prick blood was collected onto a Whatman filter card. RNA was extracted from DBS of the filter card, and fully automated multiplex PCR was performed to generate a TCR $\beta$ chain library for next generation sequencing (NGS) analysis of unique CDR3s (uCDR3).

Results: We demonstrated that the dominant clonotypes from the DBS results recapitulated those found in whole blood. According to the statistical analysis and laboratory confirmation, 40 of 2-mm punch disks from the filter cards were enough to detect the shared top clones and have strong correlation in the UCDR3 discovery with whole blood. UCDR3 discovery was neither affected by storage temperatures (room temperature versus $-20^{\circ} \mathrm{C}$ ) nor storage durations $(1,14$, and 28 days) when compared to whole blood. About 74-90\% of top $50 \mathrm{uCDR3}$ clones of whole blood could also be detected from DBS. A low rate of clonotype sharing, $0.03-1.5 \%$, was found among different individuals.

Conclusions: The DBS-based TCR repertoire profiling method is minimally invasive, provides convenient sampling, and incorporates fully automated library preparation. The system is sensitive to low RNA input, and the results are highly correlated with whole blood uCDR3 discovery allowing study scale-up to better understand the relationship and mutual influences between the immune and diseases.

Keywords: T cell receptor, TCR repertoire, CDR3, Dry blood spots, Next-generation sequence, Diversity

\section{Background}

Immunology research, particularly next generation sequencing (NGS) of the immune T-cell receptor $\beta$ $(\mathrm{TCR} \beta)$ repertoire, has advanced progression in several fields including treatment of various cancers and autoimmune diseases [1]. The advantage of immunotherapy in cancers has led to increasing numbers of studies dedicated to exploring the impact and interaction between

\footnotetext{
*Correspondence: jhan@irepertoire.com; jhan@hudsonalpha.org; sli@irepertoire.com

†'Shang-Gin Wu and Wenjing Pan contributed equally to this paper

${ }^{3}$ HudsonAlpha Institute for Biotechnology, 601 Genome Way, Huntsville, AL 35806, USA

${ }^{4}$ iRepertoire Inc., 800 Hudson Way Suite 2319, Huntsville, AL 35806, USA Full list of author information is available at the end of the article
}

immunity and cancer cells. However, the complexity of the immune system in combination with the limitation of detection methods makes this subject difficult to research.

Currently, TCR repertoire sequencing is widely used to evaluate the immune system [2]. TCR repertoires of patients were explored in a variety of disorders-patients under cancer immunotherapy, autoimmune disease [rheumatoid arthritis (RA), ankylosing spondylitis], or subject to virus infection (hepatitis, human immunodeficiency virus (HIV)) [3-7]. Analyzing the TCR repertoire may help to gain a better understanding of the immune system features and of the etiology and progression of diseases, in particular those with unknown antigenic triggers. Rapid progress has been made in the deep profiling 
of TCR repertoires by using NGS to discover millions of sequences from the TCR repertoires [8]. There are different TCR library preparation for NGS, including: multiplex PCR, targeted enrichment methods, 5'Rapid amplification of cDNA ends (5'RACE) cDNA synthesis, template-switch, and nested PCR [9]. For TCR repertoire assessment, the current standard sample type is total RNA extracted from whole blood by venipuncture. However, the costs, participant burden, regulatory constraints, and logistics associated with venipuncture and RNA handling are major barriers to clinical application or community-based research on various diseases.

Dry blood spots (DBS) have been used broadly in disease screening, drug level monitoring, and infectious microorganism detection, such as the detection of HIV and plasmodium [10,11]. There are two major advantages of this method. First, it is minimally invasive (requiring only $0.3 \mathrm{~mL}$ of capillary blood obtained by finger-or ear-prick) and a field-friendly alternative to venipuncture by professional medical staff. Second, it is low cost and convenient to handle in that the filter cards can be stored and shipped at room temperature without refrigeration for at least 1 month $[12,13]$.

The desire for TCR repertoire profiling in a variety of diseases and the advantages of a DBS-based method prompted us to establish a DBS-based TCR repertoire profiling method. Here, we report for the first time the use of a DBS sample in combination with a fully automated and closed system for TCR $\beta$ chain NGS library preparation. We have demonstrated that storage of DBS in filter cards up to 28 days at either room temperature or $-20{ }^{\circ} \mathrm{C}$ has no effect on unique CDR3 (uCDR3) discovery. Compared with whole blood, the DBS-based TCR repertoire profiling method is minimally invasive, provides convenience when sampling, is sensitive, and is highly correlated with whole blood in terms uCDR3 discovery, facilitating ease of incorporation and scale-up to studies which seek to explore the relationship between immunity in variety diseases.

\section{Materials and methods}

\section{Subjects and sample collection}

All enrolled subjects had written informed consent prior to the collection of whole blood or DBS via fingerprick. The study design and recruiting of patients were approved by the New England Independent Review Board $^{\circledR}$ (NEIRB) (IRB Number: 14-378). All experiments were performed according to the relevant guidelines and regulations.

Whatman FTA 903 cards (Sigma-Aldrich Corp. MO, USA) were used for collection of capillary blood obtained by finger-prick. In addition, peripheral blood was collected via venipuncture for comparison. The Whatman cards were stored in zip-lock plastic bags with a desiccant (ULINE. Atlanta, USA), at room temperature or $-20{ }^{\circ} \mathrm{C}$ (for longer storage) for future studies.

\section{RNA extraction from DBS and whole blood}

RNA extraction from DBS was performed following the methods proposed by Karlsson et al. [14]. 2-mm disks were punched out from a DBS with a sterile Robbins true-cut disposable 2-mm biopsy punch (Robbins Instruments, Inc., NY, USA). The punch disks were then incubated in $700 \mu \mathrm{L}$ RLT buffer plus $\beta$-mercaptoethanol $(\beta-\mathrm{ME})$. The tubes were then incubated at $37{ }^{\circ} \mathrm{C}$ for $60 \mathrm{~min}$ in a thermomixer rotating at $1000 \mathrm{rpm}$. Following incubation, RNA was extracted with a RNeasy Micro Kit (Qiagen, Valencia, CA, USA) according to the manufacturer's instruction. For whole blood, a RNeasy Mini Kit (Qiagen, Valencia, CA, USA) was used according to the manufacturer's protocol. RNA concentrations were measured by spectrophotometry.

\section{RT-PCR and sequencing of T-cell receptor $\beta$-chain CDR3 region}

iRepertoire multiplex primer sets (iRepertoire, Inc. Huntsville, AL, USA) were used to amplify the CDR3 region of TCR $\beta$ chain by using RNA as template as described by Wang et al. [15]. The whole amplification process and library preparation process for NGS were fully automated in the iR-Procecessor and iR-Cassette (iRepertoire, Inc. Huntsville, AL, USA). Then, paired-end sequencing was performed on purified PCR products using an Illumina MiSeq v2 300-cycle Reagent Kit (Illumina Inc.), for an average read depth of 30,000 reads per sample.

\section{Sequence data analysis}

Raw cDNA sequences were first analyzed to identify $\mathrm{V}$ and $\mathrm{J}$ genes by using iR-map and visualized in iRweb (iRepertoire, Inc. AL, USA). Analyzed data from iRweb include peptide sequences, alignments to the international ImMunoGeneTics (IMGT) database, uCDR3, shared CDR3s, and V- and J-gene usage. Multiple alignments and hierarchical clustering of conserved amino acid sequences were analyzed as described by Wang et al. [15].

\section{Computational error correction of bulk TCR sequences by replicates}

Errors in sequencing resulting from PCR errors, PCR contamination and read error were mitigated according to the modified method of Glanville et al.s study [16]. RNA samples were split into two reactions and processed as technical replicates. The coefficient of determination $\left(R^{2}\right)$ was calculated by linear regression to show 
the correlation between the replicates of TCR $\beta$ CDR3 frequencies prior to data analysis to exclude PCR and sequence errors.

\section{TCR repertoire diversity analysis}

The diversity of the TCR repertoire was calculated based on the diversity 50 (D50) value and the Diversity Index (DI) [17]. Briefly, D50 is the percent of dominant T cell clones that account for the cumulative $50 \%$ of the total CDR3s counted in the sample. The mathematical formulation of D50 is defined as follows:

$$
\begin{aligned}
\mathrm{D} 50= & (\text { No. of } \mathrm{uCDR} 3 \text { that make up } 50 \% \text { of the } \\
& \text { total reads } \times 100) / \text { No. of uCDR3s }
\end{aligned}
$$

The more diverse a library, the closer the value will be to 50 .

The DI is defined mathematically as follows:

Assume that the numbers (n) of uCDR3:

$$
r_{1} \geq r_{2} \geq r_{3} \geq \cdots r_{i} \geq r_{i+1} \geq \cdots \geq r_{n}
$$

where $r_{i}$ is the frequency of the $i$-th CDR3 and $n$ is the total number of unique CDR3s.

$$
x_{k}=\frac{k}{n}, \quad y_{k}=\frac{\sum_{i=1}^{k} r_{i}}{\sum_{i=1}^{n} r_{i}}
$$

In addition, tree maps and two-dimensional (2D) heat maps were used to reveal the diversity and characteristics of TCR repertoire. In a tree map, each rounded rectangle represents a unique entry: $\mathrm{V}-\mathrm{J}$ combination of uCDR3, where the size of a spot denotes the relative frequency. $2 \mathrm{D}$ heat maps showed that the relative frequency of a consensus germline V-gene allele (as per alignment with the IMGT database) is plotted relative to the consensus germline J-gene allele. Therefore, it is immediately evident which $\mathrm{V}-\mathrm{J}$ combination is used either frequently or infrequently by the color of the map.

\section{Statistical analysis}

Analysis was performed using the Statistical Package for the Social Sciences (SPSS) 17.0 software. Chi-squared test was performed on all categorical variables, with the exception of those with an expected frequency of $<5$, which were analyzed by Fisher's exact test. Two-sided Student's $t$-tests was used for comparing the means of two independent variables. One-way analysis of variance (ANOVA) was used to analyze differences in the number of uCDR3s among the different storage durations.

\section{Results}

T-cell repertoire can be detected from the extracted RNA from DBS

The work flow of the DBS-based TCR repertoire discovery is shown in Fig. 1. RNA was extracted from DBS, followed by RT-PCR in a fully automated closed system to amplify the TCR $\beta$ library. After cDNA pooling of the TCR $\beta$ library and quantification, NGS sequencing was performed, and the data was analyzed as described previously.

Since the percentage of dominant clones detected from DBS is highly dependent on TCR diversity of samples, we first calculated the number of punch disks required for dominant clone detection from whole TCR repertoire. Both confidence levels and intervals were calculated to compare TCR repertoire discovery between methods utilizing DBS versus $10 \mathrm{~mL}$ of whole blood. According to the normal range of differential count of complete blood cells, $20-35 \%$ of white blood cells are lymphocytes. $\mathrm{T}$ cells account for $70-85 \%$ of lymphocytes [18]. Therefore, there were about 700$2675 \mathrm{~T}$ cells per $\mu \mathrm{L}$ of whole blood for one man with $65 \mathrm{~kg}$ of body weight with normal white blood count range from $5000-9000 \mathrm{WBC} / \mu \mathrm{L}$. According to the Whatman card specifications, one circle with a diameter of 0.5 in. can absorb $75-80 \mu \mathrm{L}$ of blood; therefore, one 2 -mm punch disk contains approximately $2 \mu \mathrm{L}$ of blood. For individuals whose dominant clones comprise $60 \%$ of their total TCR repertoire, $80 \mu \mathrm{L}$ of blood collected on $402-\mathrm{mm}$ punch disks could provide $90 \%$ confidence level and 9\% confidence interval. The volume of blood required for a given confidence level and interval is presented in Additional file 1: Table S1.

Having calculated the punch disk numbers required for TCR repertoire discovery, we compared between $3,10,40$ and 80 punch disks. The average uCDR3 discovery for $3,10,40$, and 80 disks were 169, 246, 1726, and 1581, respectively, with an average read depth of 30,000 reads per sample. The uCDR3 discovery from 40 disks was higher than those from $3(p<0.0001)$ or 10 punch disks $(p<0.0001)$. There was no significant statistical difference in average uCDR3 numbers between 40 and 80 punch disks $(p=0.520)$ (Fig. 2a). The tree maps and heat maps also revealed that the diversity and the identified combination of $\mathrm{V}$ - and $\mathrm{J}$ - gene segments were obviously higher in 40 or 80 punch disks than those in 3 or 10 punch disks (Fig. 2b, c). As a result, 40 disks were established as the optimum punch number for future studies. 


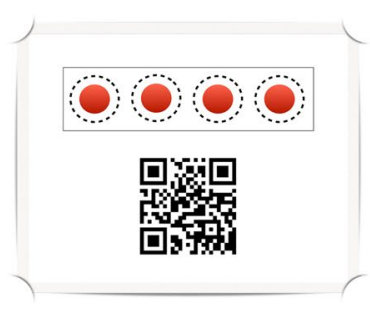

DBS Filter Card

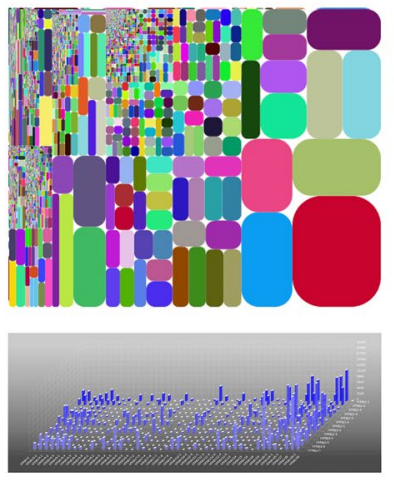

Data Analysis

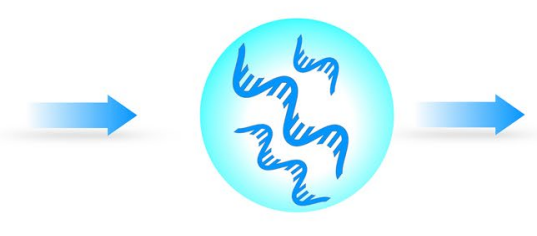

RNA

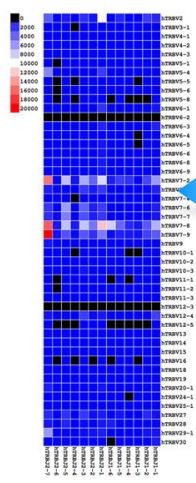

munun!

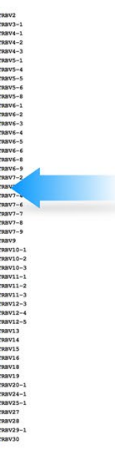

.

$$
\text { ๑. }
$$

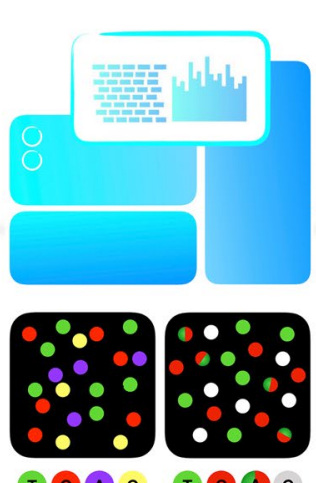

NGS

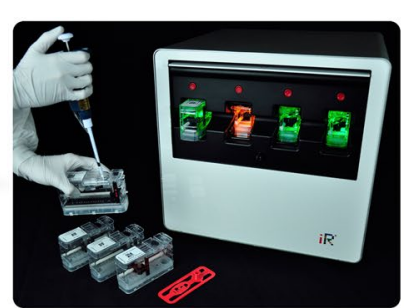

\section{Library} Preparation

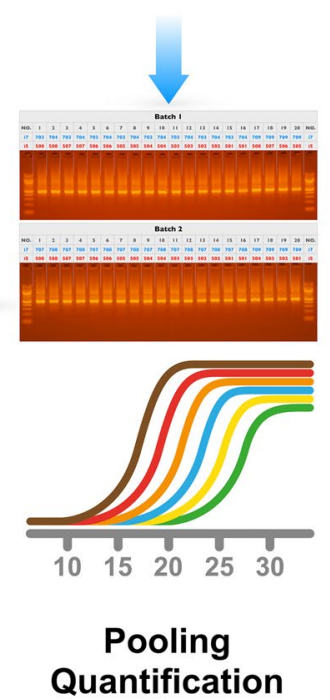

Fig. 1 The work flow of DBS based TCR repertoire discovery. Finger-prick blood is collected onto the filter card and extracted RNA from DBS is used as a template for amplification. The entire PCR amplification and library preparation are automated in a closed cassette. Post amplification, libraries are pooled and quantified then sequenced by NGS. Sequencing data is analyzed by iRweb's bioinformatics platform

CDR3 frequency correlation between DBS and whole blood The $R^{2}$ of uCDR3 frequencies were calculated by linear regression between technical replicates to exclude PCR or sequencing error. The $R^{2}$ of the regression for CDR3 discovery between technical replicates for DBS and whole blood samples were 0.990 and 0.995 , respectively (Fig. 3a-d). uCDR3 frequency correlations were then determined to compare discovery between DBS and whole blood samples. uCDR3 discovery between DBS and whole blood revealed high regression correlation $\left(R^{2}=0.986\right.$ and $R^{2}=0.987$, respectively) (Fig. 3e), with $94 \%$ and $92 \%$ of the top 50 and top 100 dominant clones from whole blood being detected from DBS, respectively (data not shown).

\section{Effect of storage temperature and duration on uCDR3 discovery}

Despite the manufacturer's suggested storage temperature of $-20^{\circ} \mathrm{C}$, prior studies show conflicting results for the effect of DBS storage temperature and duration on RNA preservation [19-22]. In this study, we compared the uCDR3 discovery between two storage temperatures (room temperature and $-20{ }^{\circ} \mathrm{C}$ ). No significant difference was demonstrated in uCDR3 discovery between DBS samples stored at room temperature and $-20^{\circ} \mathrm{C}$ for 14 days $(p=0.922)$ or 28 days $(p=0.700)$ (Fig. 4$)$.

uCDR3 discovery from storage duration tests (1 day, 14 days and 28 days) was also compared in DBS samples with low, moderate, and high CDR3 diversities. Among different storage durations, no significant differences were found in uCDR3 discovery in DBS samples with low $(p=0.190)$, moderate $(p=0.077)$, and high $(p=0.857)$ uCDR3 diversities (Fig. 5), and this is true even under the two different storage temperatures tested, room temperature, and $-20^{\circ} \mathrm{C}$ (Fig. 4). The uCDR3 frequency did not decrease over time. These results indicate that storage duration of 1,14 and 28 days had no impact on uCDR3 discovery. 


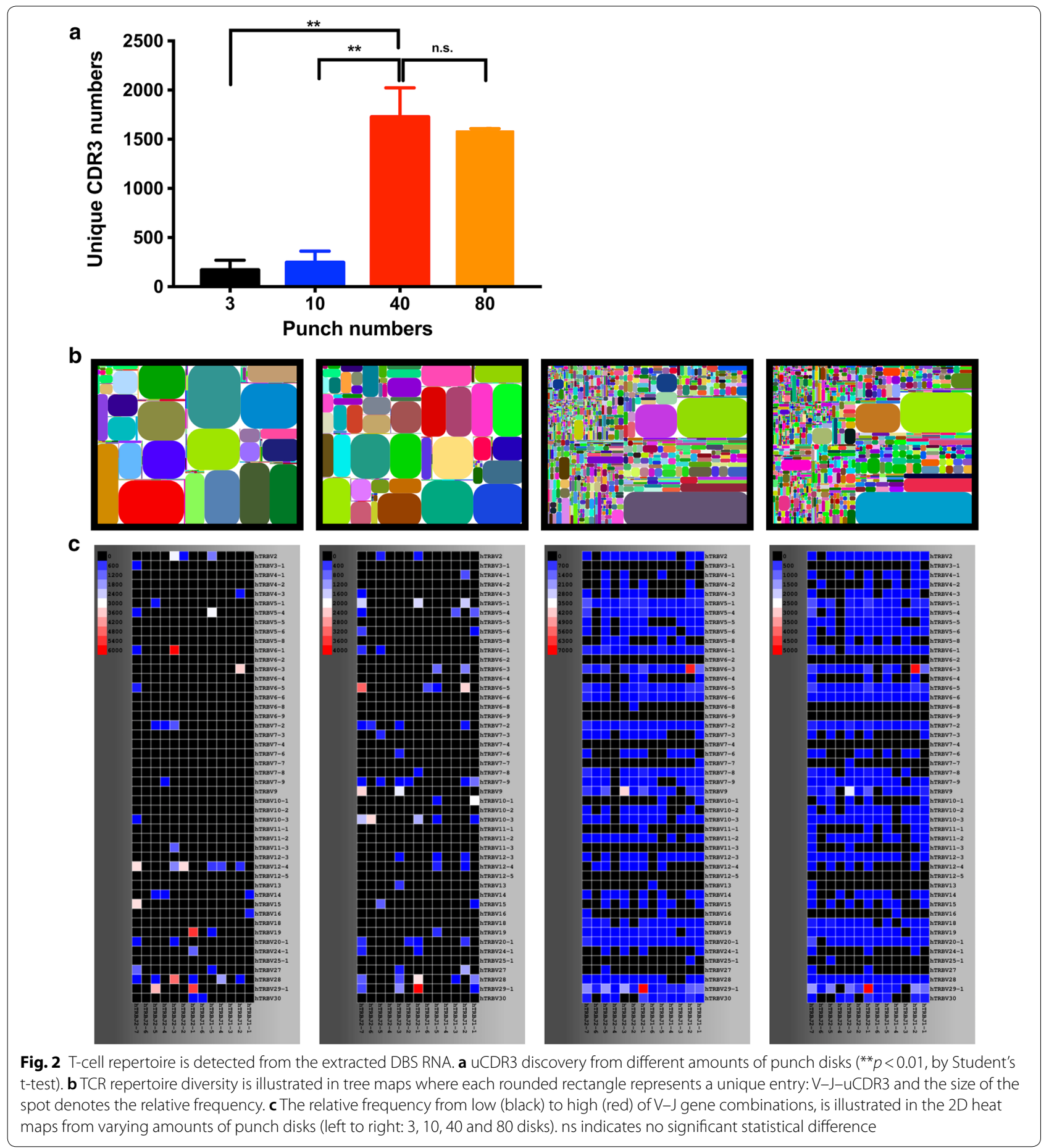

We also demonstrated the relationship of TCR $\beta$ clonotype discovery between DBS and whole blood (Fig. 6). The $R^{2}$ of the regression for CDR3 discovery between technical replicates for whole blood samples and DBS in different storage duration tests were 0.990 and $0.86-0.95$, respectively. Long storage duration up to 28 days not only showed no impact on uCDR3 discovery, but also showed no impact on the correlation uCDR3 discovery between DBS and whole blood. In addition, the top 50 and 100 dominant CDR3 clones of whole blood could be detected 

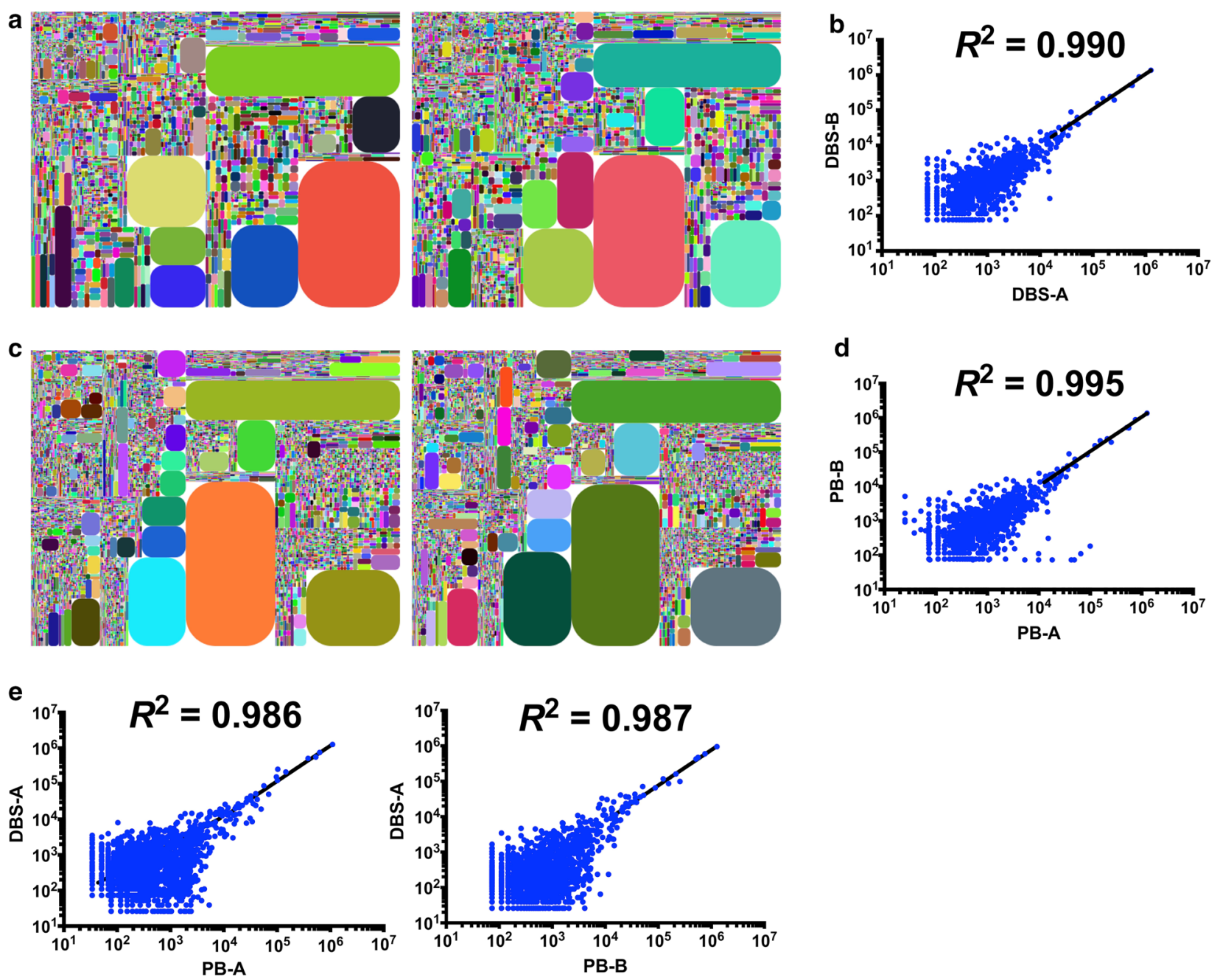

Fig. 3 The comparison of TCR $\beta$ CDR3 between DBS and whole blood. a Tree maps of TCR $\beta$ CDR3 from duplicate DBS samples. $\mathbf{b}$ The coefficient of determination $\left(R^{2}\right)$ of linear regression in TCRB CDR3 sequences from duplicate DBS samples. $\mathbf{c}$ Tree maps of TCR 3 CDR3 from duplicate peripheral blood samples. $\mathbf{d}$ The $R^{2}$ of linear regression in TCR $\beta$ CDR3 sequences from duplicate peripheral blood samples. e The $R^{2}$ of linear regression in TCR $\beta$ CDR3 sequences between peripheral blood and DBS

by DBS for $62-88 \%$ and $45-69 \%$ of the dominant unique CDR3s at 28-day storage, respectively (Fig. 6).

\section{Relationship between TCR repertoire diversity and uCDR3 discovery}

The diversity of each sample's TCR repertoire can impact the correlation of uCDR3 discovery between DBS and whole blood samples. To explore this relationship, uCDR3 discovery was compared between DBS and whole blood samples known to have low, moderate, and high diversities of TCR repertoire. Table 1 shows the characteristics of TCR $\beta$ CDR3 between DBS and whole blood. The samples with low and moderate TCR diversity have a higher regression correlation between DBS and whole blood samples than those with higher TCR diversity $\left(R^{2}=0.989\right.$ for low diversity, 0.865 for moderate diversity and 0.806 for high diversify). $92 \%$ and $87 \%$ of the top 100 uCDR3 clones from whole blood could be detected in a DBS sample of low and moderate diversity, respectively, but only $74 \%$ of those in high diversity samples. For samples with high TCR diversity, the top 50 uCDR3 clones from whole blood could still be detected for $82 \%$ in a DBS sample. Although the diversity of samples had impact on the correlation of uCDR3 discovery, there was still a relatively high amount of shared uCDR3s between DBS sample and whole blood sample. 


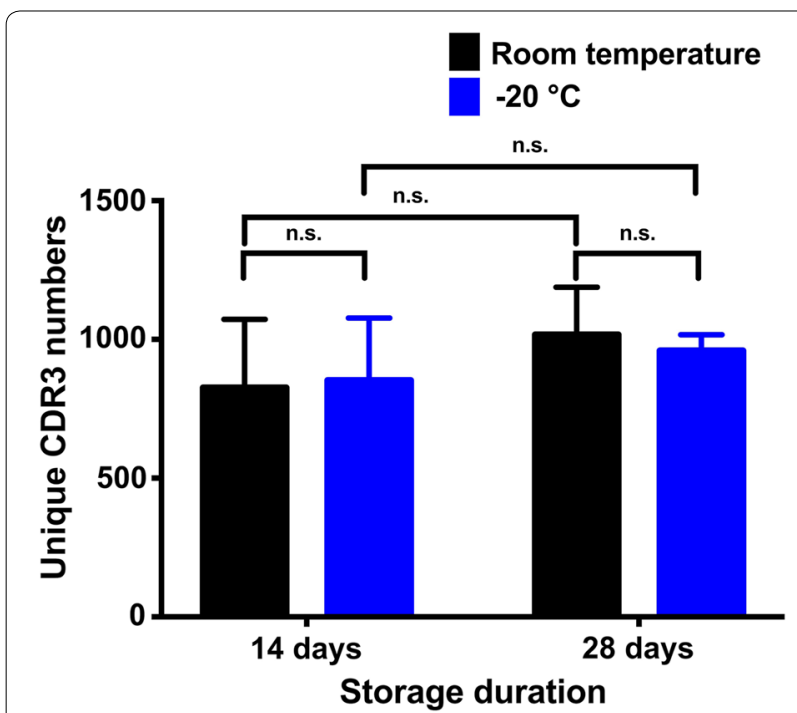

Fig. 4 The comparison of UCDR3 discovery between different storage temperatures (room temperature versus $-20^{\circ} \mathrm{C}$ ). Student's $t$ test was used to determine that there was no significant difference in UCDR3 discovery between the two storage temperatures at both 14 days ( $p=0.922$ ) and 28 days $(p=0.700)$ storage duration. ns indicates no significant statistic difference

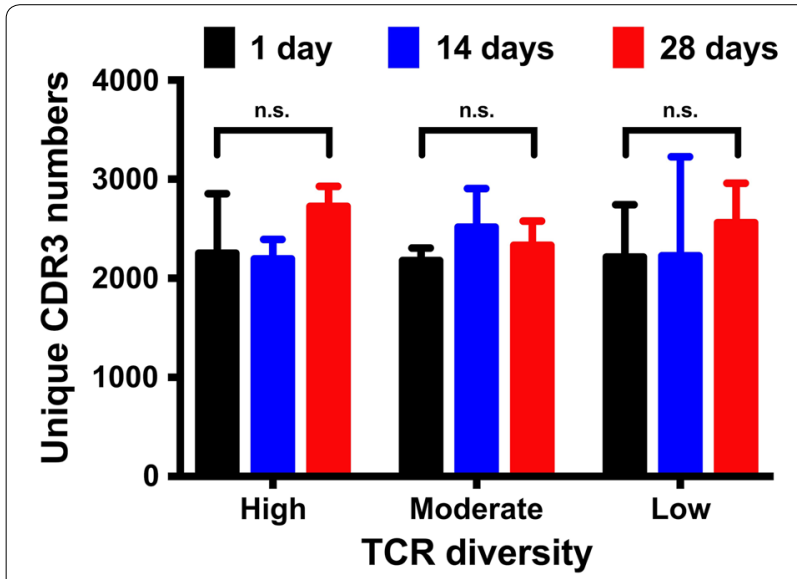

Fig. 5 Comparison of UCDR3 discovery with storage durations of 1, 14 and 28 days from DBS samples with varying TCR diversities. There was no significant difference in TCR $\beta$ UCDR3 discovery between storage durations for samples with varying TCR diversities by one-way ANOVA analysis. ns indicates no significant statistic difference

\section{uCDR3 sharing between samples}

In order to determine whether the TCR $\beta$ CDR3s detected by this method are unique across individuals, the TCR $\beta$ CDR3 sharing was compared among different individuals. The sharing rate of $\mathrm{uCDR} 3$ between two individuals was low, ranging from $0.03 \%$ to $1.55 \%$ (Table 2 and Additional file 1: Table S2). The uCDR3 sharing rate between
P5 and P6 is the highest; however, this is a parent-child relationship.

\section{Discussion}

In this study, we reported for the first time the establishment of a DBS-based method as a minimally invasive alternative to venipuncture blood sampling for TCR repertoire studies. In addition to its cost effectiveness, we have demonstrated that the storage temperature and storage duration had no effect on uCDR3 discovery. The ease of sample collection and the convenience of storage for transportation purposes makes it a viable option for scale-up studies related to TCR repertoires.

The FTA cards entrap nucleic acids and are stabilized at room temperature, which allows long-term storage [20, 23, 24]. DNA extracted from the stored card yields adequate quality and amount for PCR amplification compared with that extracted from the fresh samples [25, 26]. The extracted DNA from the card can also be used for high-throughput molecular analysis [13, 27]. In addition, DBS also provides an alternative tool to formalin-fixed paraffin-embedded cell blocks for biobanking to detect epidermal growth factor receptor $(E G F R)$ or Kirsten rat sarcoma 2 viral oncogene homolog (KRAS) mutation for personalized target therapy in lung cancer patients [28]. Therefore, this method can be utilized in multiple fields, not only cancer diagnosis but various disease etiologies. In addition to the stability of high-quality nucleic acids, the low cost of storage, and ease in transportation, FTA cards serve as an alternative to various traditional storage methods [20].

We established a method that uses RNA extracted from DBS for high-throughput sequencing. The entire process of RT-PCR amplification and library preparation of the immune repertoire was conducted automatically in cassettes. Automated amplification in a closed, contained environment throughout the entire process prevents contamination and improves the consistency of the results by avoiding error or variation, which may be introduced during manual processing. TCR repertoires derived from DBS are directly comparable with the dominant clones of TCR repertoire discovery from whole blood. As a result, DBS may be used as a screening tool for certain diseases or for genetic screening instead of whole blood.

Peluso et al. used FTA cards to store fine needle aspiration cytology samples of lymph node, and they amplified immunoglobulin heavy and light chains, TCR- $\beta$ and $\gamma$ chains by PCR from DNA [29]. The results showed that the amplified DNA from FTA cards is comparable to those of cryopreserved samples. However, a tremendous diversity of TCR repertoires results from extensive recombination, splicing and post-transcriptional processing to yield functional proteins. TCR repertoire 


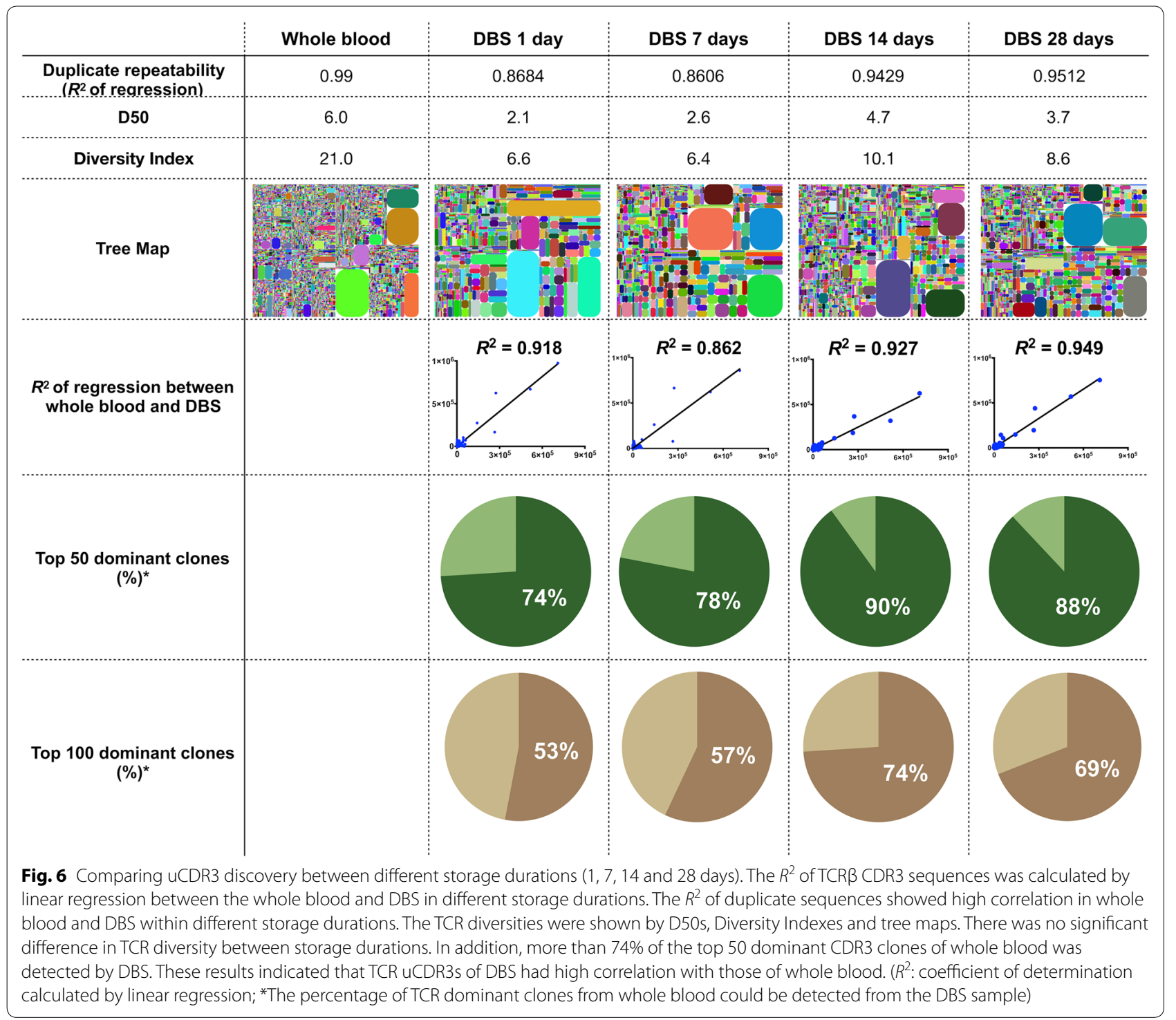

Table 1 The clinical characteristics of samples with low, moderate and high TCR repertoire diversities

\begin{tabular}{|c|c|c|c|c|c|c|}
\hline \multirow{2}{*}{$\begin{array}{l}\text { Diversity } \\
\text { Sample }\end{array}$} & \multicolumn{2}{|l|}{ Low } & \multicolumn{2}{|c|}{ Moderate } & \multicolumn{2}{|l|}{ High } \\
\hline & WB & DBS & WB & DBS & WB & DBS \\
\hline Duplicate sequence repeatability $\left(R^{2}\right)$ & 0.994 & 0.991 & 0.993 & 0.956 & 0.930 & 0.699 \\
\hline Unique CDR3 & 43,653 & 5327 & 38,431 & 3820 & 25,118 & 3208 \\
\hline D50 & 0.3 & 1.1 & 2.7 & 7.6 & 14.5 & 6.8 \\
\hline Diversity Index & 15.8 & 13.2 & 20.6 & 16.8 & 27.1 & 11.1 \\
\hline$R^{2}$ of regression between DBS and WB & & 0.989 & & 0.865 & & 0.806 \\
\hline Top 50 dominant clones (\%) & & $94 \%$ & & $98 \%$ & & $82 \%$ \\
\hline Top 100 dominant clones (\%) ${ }^{\mathrm{a}}$ & & $92 \%$ & & $87 \%$ & & $74 \%$ \\
\hline
\end{tabular}

WB whole blood, $D B S$ dry blood spot, $R^{2}$ coefficient of determination calculated by linear regression

a The percentage of TCR dominant clones from whole blood could be found from DSB examination 
Table 2 The ratio of the shared UCDR3 reads divided by the total reads of the individual

\begin{tabular}{|c|c|c|c|c|c|c|c|}
\hline & P1 & P2 & P3 & P4 & P5 & P6 & P7 \\
\hline P1 & & $0.03 \%$ & $0.35 \%$ & $0.08 \%$ & $0.00 \%$ & $0.05 \%$ & $0.03 \%$ \\
\hline P2 & $0.07 \%$ & & $0.24 \%$ & $0.05 \%$ & $0.06 \%$ & $0.14 \%$ & $0.00 \%$ \\
\hline P3 & $0.30 \%$ & $0.09 \%$ & & $0.15 \%$ & $0.41 \%$ & $0.08 \%$ & $0.88 \%$ \\
\hline P4 & $0.27 \%$ & $0.10 \%$ & $0.68 \%$ & & $0.13 \%$ & $0.10 \%$ & $0.31 \%$ \\
\hline P5 & $0.01 \%$ & $0.12 \%$ & $0.30 \%$ & $0.00 \%$ & & $0.32 \%$ & $0.68 \%$ \\
\hline P6 & $0.03 \%$ & $0.22 \%$ & $0.34 \%$ & $0.62 \%$ & $1.55 \%$ & & $1.10 \%$ \\
\hline P7 & $0.09 \%$ & $0.00 \%$ & $0.83 \%$ & $0.10 \%$ & $0.08 \%$ & $0.20 \%$ & \\
\hline
\end{tabular}

discovery from RNA rather than genomic DNA prevents the inclusion of nonproductive or functionally irrelevant sequences [30]. There is currently no study report on the direct detection of the TCR $\beta$ repertoire from fingerpricked blood stored on FTA cards.

Although it is known that high-quality DNA can be extracted from the FTA cards [25, 31], there are few studies about RNA extracted from FTA cards compared with other preservation methods. Instability of RNA is a major concern with storage, temperature, and extraction methods. The World Health Organization (WHO) suggests that for HIV drug resistance, dried blood spot specimens should be transferred to $-20^{\circ} \mathrm{C}$ or lower as soon as possible although they can be kept and/or transported at ambient temperature up to 14 days after collection [10]. However, Bertagolio et al. demonstrated that a $90 \%$ positive amplification rate was noted under the ambient storage temperature of DBS [19]. This is compatible with the current DBS study which shows comparable numbers of uCDR3s between disparate storage temperatures of room temperature and $-20{ }^{\circ} \mathrm{C}$, even after 28 days of storage duration. Therefore, DBS can be stored and transported at room temperature without impacting TCR $\beta$ clonotype discovery.

Approximately $74-98 \%$ of the top 50 dominant clones from the whole blood are found in the DBS sample. This result indicates that the DBS-based immune repertoire profiling is very sensitive. According to the statistical analysis of sample sizes and the results of the current study, we are now confident that our DBS-based method can reflect whole blood TCR $\beta$ repertoire sampling with very good coverage and high sensitivity, i.e., enough shared top clonotypes and strong correlation in uCDR3 discovery.

The recent technological advances in the fields of immunology and immunotherapy hold promise for patients with cancers [32-34]. The TCR repertoire plays a pivotal factor in immunity [35]. For instance, TCR repertoire profiling has the potential to serve as a biomarker of treatment response in pancreatic ductal carcinoma patients who received immunotherapy [7]. Through recognition of major histocompatibility complex (MHC)peptide complex of TCRs, T cells can be activated and specific $\mathrm{T}$ cell clones expanded to give a response to foreign pathogens or cancer cells [36].

Accumulating evidence suggests that immune cells play pivotal roles in a variety diseases and their overlapping regulatory mechanisms in addition to cancers, and TCR repertoire were also explored in different area. For example: Muraro et al. used TCR repertoire to follow the effect of autologous stem cell transplantation in multiple sclerosis patients [37]. For autoimmune diseases, the median TCR $\beta$-chain frequency in RA patients was increased tenfold, indicating marked contraction of the repertoire, and 
disease activity of RA was negatively correlated with the TCR repertoire diversity of CD4+ T cells [4,38]. Even for infection disease, TCR repertoire was explored the function profile of T cells in HIV patients and the characteristics of immune landscape after injection of HIV vaccine $[6,39]$. TCR $\beta$-CDR3 were correlated with disease prognosis of hepatitis B and C [5, 40, 41]. DBS-based methodology provides a simple and quick approach to access changes of immunity and treatment effect in different disease areas.

Each individual has more than $10^{9} \mathrm{~T}$ cells which express unique heterodimeric $\mathrm{T}$-cell receptors as identified by the CDR3 peptide sequence of the TCR $\beta$ chain. This high diversity provides defense against foreign pathogens. Hou et al. reported that on average two individuals can share $4.85 \pm 2.5 \%$ of their DNA sequences and $12.17 \pm 0.81 \%$ of their CDR3 amino acid sequences [42]. According to the current study, less than $1 \%$ of uCDR3 clones are shared between two different people except when a parent-child relationship exists (Table 2). Putintseva et al. reported that the degree of overlap was always slightly higher for related individuals for all CDR3s, but this difference never approached a significant level compared to unrelated individuals [43]. In a parent-child relationship, $50 \%$ of their HLA alleles are shared. Exposure to the same pathogens may result in specific $T$ cell expansion. Because V-gene usage is highly influenced by human leukocyte antigen (HLA)-type, certain V-genes may be preferentially selected by the immune system, ultimately allowing more shared CDR3s among closely related individuals. The high individual specificity detected by this method allows researchers to look for public CDR3s in a homogenous group or identify disease signatures, based on shared unique CDR3 peptides.

Figure 7 demonstrates the advantages of the DBSbased method for TCR repertoire analysis. DBS provides a minimally invasive sample collection alternative to venipuncture. It is convenient for storage and transportation, in contrast to the necessary of refrigeration of $-20^{\circ} \mathrm{C}$ for some RNA-compatible vacutainer tubes, and cold chain shipping for whole blood. Then, high quality RNA can be extracted from DBS-based samples for future molecular amplification and analysis. The entire process of RTPCR amplification and library preparation of the immune repertoire was conducted automatically in a fully closed cassette, which is easier than the labor-intensive manual process. It also helps improve result consistency and to prevent contamination of the laboratory environment with amplicon. Although other products (PAXgene Blood RNA Tube (IVD) (Qiagen), Tempus ${ }^{\mathrm{TM}}$ Blood RNA Tube, etc.) can be used for storage and transportation of RNA at room temperature, refrigeration is still necessary for long-term storage. In addition, they are more expensive and laborious than DBS-based processing due to the requirement for a phlebotomist and specialized extraction RNA kits.

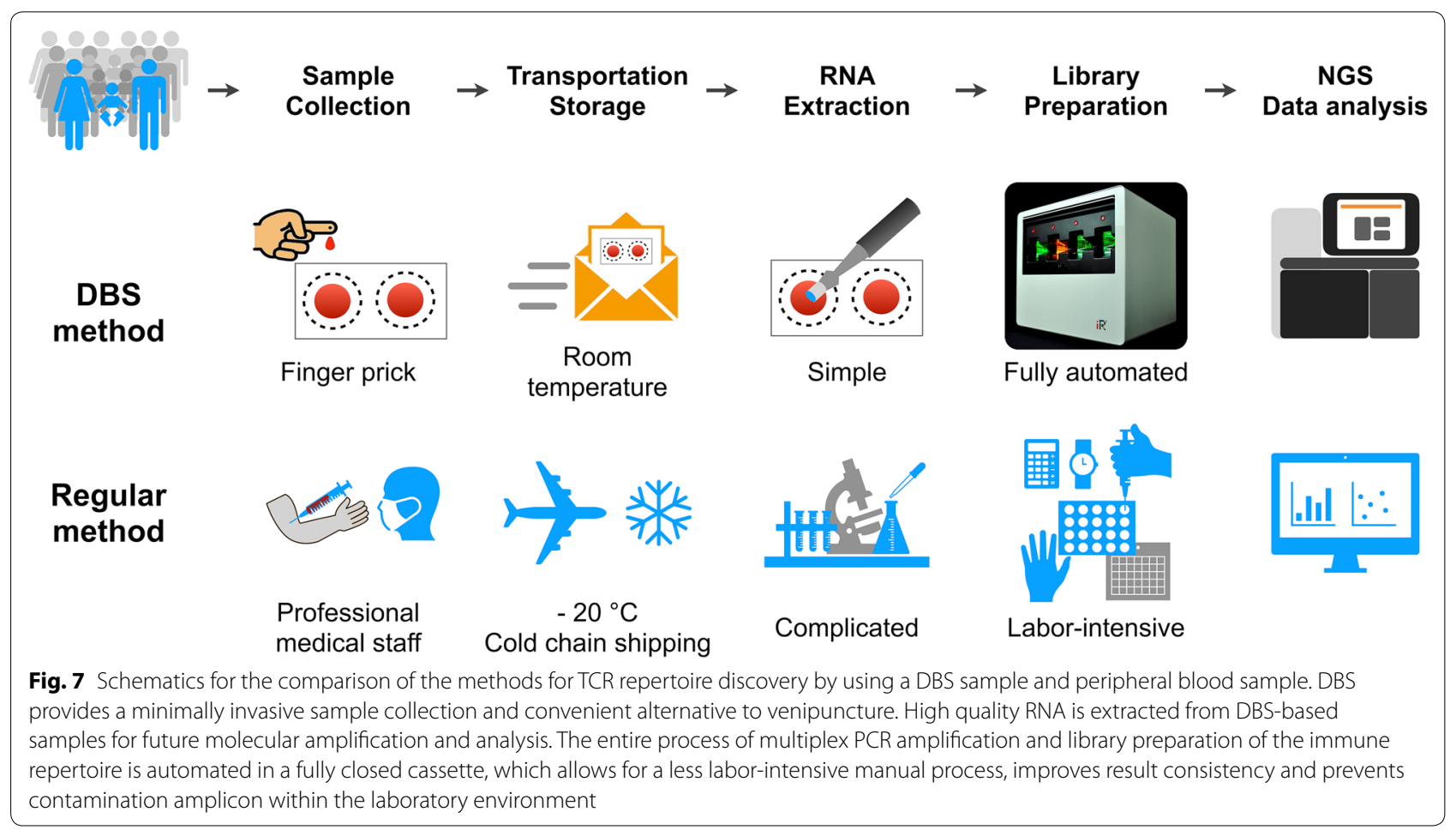




\section{Conclusions}

The current DBS-based method shows high correlation to dominant TCR $\beta$ CDR3 clones detected from whole blood. The minimally invasive, cost effective, and convenient storage conditions will allow for the scale-up of surveillance studies or treatment response. More importantly, the development of such a tool allowed collecting of real-world data, i.e., when symptoms showed up, rather than doctors available for an appointment.

\section{Additional file}

Additional file 1: Table S1. The statistical results of sample (blood volume $(\mu \mathrm{L})$ ) calculation; Table S2. The numbers of shared CDR3 among the different people.

\section{Abbreviations}

NGS: next generation sequencing; TCR: T-cell receptor; 5'RACE: 5'Rapid amplification of cDNA ends; DBS: dry blood spots; RA: rheumatoid arthritis; HIV: human immunodeficiency virus; UCDR3: unique CDR3s; $\beta-M E$ : $\beta$-mercaptoethanol; IMGT: ImMunoGeneTics; DI: Diversity Index; $R^{2}$ : the coefficient of determination; 2D: two-dimensional; SPSS: Statistical Package for the Social Sciences; ANOVA: one-way analysis of variance; EGFR: epidermal growth factor receptor; KRAS: Kirsten rat sarcoma 2 viral oncogene homolog; WHO: The World Health Organization; MHC: major histocompatibility complex.

\section{Authors' contributions}

SW, SL, JH and WP designed the study and patient specimen collection. SW, SL and WP completed the experimental process, data collection, literature search and generation of figures. SW, SL, XH, MBS; BB and MD wrote and edited the manuscript. $\mathrm{HX}$ and $\mathrm{NH}$ provided the experimental technical and laboratory facilities support. All authors reviewed the manuscript. All authors read and approved the final manuscript.

\section{Author details}

1 Department of Internal Medicine, National Taiwan University Hospital, National Taiwan University, Taipei 10002, Taiwan. ${ }^{2}$ Department of Internal Medicine, National Taiwan University Cancer Center, National Taiwan University, Taipei 10672, Taiwan. ${ }^{3}$ HudsonAlpha Institute for Biotechnology, 601 Genome Way, Huntsville, AL 35806, USA. ${ }^{4}$ iRepertoire Inc., 800 Hudson Way Suite 2319, Huntsville, AL 35806, USA. ${ }^{5}$ iCubate Inc., Huntsville, AL 35806, USA.

\section{Acknowledgements}

Not applicable.

\section{Competing interests}

WP, MBS, BB, MD, XH, SL, and JH are employees of iRepertoire. The other authors declare no competing interests.

\section{Availability of data and materials}

The data and materials supporting the conclusions of the current study are available from the corresponding author on reasonable request.

\section{Consent for publication}

Not applicable.

\section{Ethics approval and consent to participate}

The study design and recruiting of patients were approved by the New England Independent Review Board ${ }^{\circledR}$ (NEIRB) (IRB Number: 14-378). Written informed consent was obtained from all study participants. All experiments were performed according to the relevant guidelines and regulations.

\section{Funding}

The study did not receive any funding support.

\section{Publisher's Note}

Springer Nature remains neutral with regard to jurisdictional claims in published maps and institutional affiliations.

Received: 8 January 2019 Accepted: 9 February 2019

Published online: 18 February 2019

\section{References}

1. Davis MM, Tato CM, Furman D. Systems immunology: just getting started. Nat Immunol. 2017;18:725-32.

2. Ichinohe T, Miyama T, Kawase T, Honjo Y, Kitaura K, Sato H, et al. Nextgeneration immune repertoire sequencing as a clue to elucidate the landscape of immune modulation by host-gut microbiome interactions. Front Immunol. 2018;9:668.

3. Cui JH, Jin YB, Lin KR, Xiao P, Chen XP, Pan YM, et al. Characterization of peripheral blood TCR repertoire in patients with ankylosing spondylitis by high-throughput sequencing. Hum Immunol. 2018;79:485-90.

4. Sakurai K, Ishigaki K, Shoda H, Nagafuchi Y, Tsuchida Y, Sumitomo S, et al. HLA-DRB1 shared epitope alleles and disease activity are correlated with reduced $T$ cell receptor repertoire diversity in CD4+ T cells in rheumatoid arthritis. J Rheumatol. 2018;45:905-14

5. Ping $Y$, Song $M$, Wang M, Li Z, Zhang Y. CDR3 repertoire diversity of CD8+ T lymphocytes in patients with HCV. Cell Immunol. 2018;56:56. https:// doi.org/10.1016/j.cellimm.2018.12.007 (Epub ahead of print).

6. Wendel BS, Del Alcazar D, He C, Del Rio-Estrada PM, Aiamkitsumrit $\mathrm{B}$, Ablanedo-Terrazas $\mathrm{Y}$, et al. The receptor repertoire and functional profile of follicular T cells in HIV-infected lymph nodes. Sci Immunol. 2018;3:eaan8884. https://doi.org/10.1126/sciimmunol.aan8884.

7. Hopkins AC, Yarchoan M, Durham JN, Yusko EC, Rytlewski JA, Robins HS, et al. T cell receptor repertoire features associated with survival in immunotherapy-treated pancreatic ductal adenocarcinoma. JCI Insight. 2018;3. https://doi.org/10.1172/jci.insight.122092.

8. Benichou J, Ben-Hamo R, Louzoun Y, Efroni S. Rep-Seq: uncovering the immunological repertoire through next-generation sequencing. Immunology. 2012;135:183-91.

9. Rosati E, Dowds CM, Liaskou E, Henriksen EKK, Karlsen TH, Franke A. Overview of methodologies for T-cell receptor repertoire analysis. BMC Biotechnol. 2017;17:61.

10. WHO. WHO manual for HIV drug resistance testing using dried blood spot specimens. Geneva: WHO; 2010.

11. Zainabadi K, Adams M, Han ZY, Lwin HW, Han KT, Ouattara A, et al. A novel method for extracting nucleic acids from dried blood spots for ultrasensitive detection of low-density Plasmodium falciparum and Plasmodium vivax infections. Malar J. 2017;16:377.

12. Rahikainen AL, Palo JU, de Leeuw W, Budowle B, Sajantila A. DNA quality and quantity from up to 16 years old post-mortem blood stored on FTA cards. Forensic Sci Int. 2016;261:148-53.

13. Saieg MA, Geddie WR, Boerner SL, Liu N, Tsao M, Zhang T, et al. The use of FTA cards for preserving unfixed cytological material for high-throughput molecular analysis. Cancer Cytopathol. 2012;120:206-14.

14. Karlsson H, Guthenberg C, von Dobeln U, Kristenssson K. Extraction of RNA from dried blood on filter papers after long-term storage. Clin Chem. 2003;49:979-81.

15. Wang C, Sanders CM, Yang Q, Schroeder HW Jr, Wang E, Babrzadeh F, et al. High throughput sequencing reveals a complex pattern of dynamic interrelationships among human T cell subsets. Proc Natl Acad Sci USA. 2010;107:1518-23.

16. Glanville J, Huang H, Nau A, Hatton O, Wagar LE, Rubelt F, et al. Identifying specificity groups in the T cell receptor repertoire. Nature. 2017;547:94-8.

17. Han J. Immunodiversity assessment method and its use. US20120183969 USA; 2012.

18. Chernecky CC, Berger BJ, Laboratory tests and diagnostic procedures. 6th ed. In: Chernecky CC, Berger BJ, editors. St. Louis: Elsevier; 2013. p. 432-59. 
19. Bertagnolio S, Soto-Ramirez L, Pilon R, Rodriguez R, Viveros M, Fuentes L, et al. HIV-1 drug resistance surveillance using dried whole blood spots. Antivir Ther. 2007;12:107-13.

20. da Cunha Santos G. FTA cards for preservation of nucleic acids for molecular assays: a review on the use of cytologic/tissue samples. Arch Pathol Lab Med. 2018;142:308-12.

21. Garcia-Lerma JG, McNulty A, Jennings C, Huang D, Heneine W, Bremer JW. Rapid decline in the efficiency of HIV drug resistance genotyping from dried blood spots (DBS) and dried plasma spots (DPS) stored at $37^{\circ} \mathrm{C}$ and high humidity. J Antimicrob Chemother. 2009;64:33-6.

22. Nelson JA, Loftis AM, Kamwendo D, Fawzi WW, Taha TE, Goldenberg $R L$, et al. Nevirapine resistance in human immunodeficiency virus type 1-positive infants determined using dried blood spots stored for up to six years at room temperature. J Clin Microbiol. 2009;47:1209-11.

23. Barth H, Morel A, Mougin C, Averous G, Legrain M, Fender M, et al. Longterm storage and safe retrieval of human papillomavirus DNA using FTA elute cards. J Virol Methods. 2016;229:60-5

24. Rajendram D, Ayenza R, Holder FM, Moran B, Long T, Shah HN. Long-term storage and safe retrieval of DNA from microorganisms for molecular analysis using FTA matrix cards. J Microbiol Methods. 2006:67:582-92.

25. Peluso AL, Cascone AM, Lucchese L, Cozzolino I, leni A, Mignogna C, et al. Use of FTA cards for the storage of breast carcinoma nucleic acid on fineneedle aspiration samples. Cancer Cytopathol. 2015;123:582-92.

26. Dobbs LJ, Madigan MN, Carter AB, Earls L. Use of FTA gene guard filter paper for the storage and transportation of tumor cells for molecular testing. Arch Pathol Lab Med. 2002;126:56-63.

27. Kampmann ML, Buchard A, Borsting C, Morling N. High-throughput sequencing of forensic genetic samples using punches of FTA cards with buccal swabs. Biotechniques. 2016:61:149-51.

28. da Cunha Santos G, Liu N, Tsao MS, Kamel-Reid S, Chin K, Geddie WR. Detection of EGFR and KRAS mutations in fine-needle aspirates stored on Whatman FTA cards: is this the tool for biobanking cytological samples in the molecular era? Cancer Cytopathol. 2010;118:450-6.

29. Peluso AL, Cozzolino I, Bottiglieri A, Lucchese L, Di Crescenzo RM, Langella $\mathrm{M}$, et al. Immunoglobulin heavy and light chains and T-cell receptor beta and gamma chains PCR assessment on cytological samples. A study comparing FTA cards and cryopreserved lymph node fine-needle cytology. Cytopathology. 2017:28:203-15.

30. Calis JJ, Rosenberg BR. Characterizing immune repertoires by high throughput sequencing: strategies and applications. Trends Immunol. 2014;35:581-90.
31. McClure MC, McKay SD, Schnabel RD, Taylor JF. Assessment of DNA extracted from FTA cards for use on the Illumina iSelect BeadChip. BMC Res Notes. 2009:2:107.

32. Kaufman HL, Kirkwood JM, Hodi FS, Agarwala S, Amatruda T, Bines SD, et al. The Society for Immunotherapy of Cancer consensus statement on tumour immunotherapy for the treatment of cutaneous melanoma. Nat Rev Clin Oncol. 2013:10:588-98.

33. Robert L, Harview C, Emerson R, Wang X, Mok S, Homet B, et al. Distinct immunological mechanisms of CTLA-4 and PD-1 blockade revealed by analyzing TCR usage in blood lymphocytes. Oncoimmunology. 2014:3:e29244.

34. Robert L, Tsoi J, Wang X, Emerson R, Homet B, Chodon T, et al. CTLA4 blockade broadens the peripheral T-cell receptor repertoire. Clin Cancer Res. 2014:20:2424-32.

35. Cooper MD, Alder MN. The evolution of adaptive immune systems. Cell. 2006;124:815-22

36. Castro CD, Luoma AM, Adams EJ. Coevolution of T-cell receptors with MHC and non-MHC ligands. Immunol Rev. 2015;267:30-55.

37. Muraro PA, Robins H, Malhotra S, Howell M, Phippard D, Desmarais C, et al. T cell repertoire following autologous stem cell transplantation for multiple sclerosis. J Clin Invest. 2014;124:1168-72.

38. Wagner UG, Koetz K, Weyand CM, Goronzy JJ. Perturbation of the T cell repertoire in rheumatoid arthritis. Proc Natl Acad Sci USA. 1998;95:14447-52.

39. Wang L, Zhang W, Lin L, Li X, Saksena NK, Wu J, et al. A comprehensive analysis of the $T$ and $B$ lymphocytes repertoire shaped by HIV vaccines. Front Immunol. 2018:9:2194.

40. Jiang Q, Zhao T, Zheng W, Zhou J, Wang H, Dong H, et al. Patient-shared TCRbeta-CDR3 clonotypes correlate with favorable prognosis in chronic hepatitis B. Eur J Immunol. 2018;48:1539-49.

41. Yang G, Ou M, Chen H, Guo C, Chen J, Lin H, et al. Characteristic analysis of TCR beta-chain CDR3 repertoire for pre- and post-liver transplantation. Oncotarget. 2018:9:34506-19.

42. Hou X, Wang M, Lu C, Xie Q, Cui G, Chen J, et al. Analysis of the repertoire features of TCR beta chain CDR3 in human by high-throughput sequencing. Cell Physiol Biochem. 2016;39:651-67.

43. Putintseva EV, Britanova OV, Staroverov DB, Merzlyak EM, Turchaninova MA, Shugay M, et al. Mother and child T cell receptor repertoires: deep profiling study. Front Immunol. 2013;4:463
Ready to submit your research? Choose BMC and benefit from:

- fast, convenient online submission

- thorough peer review by experienced researchers in your field

- rapid publication on acceptance

- support for research data, including large and complex data types

- gold Open Access which fosters wider collaboration and increased citations

- maximum visibility for your research: over 100M website views per year

At BMC, research is always in progress.

Learn more biomedcentral.com/submissions 